\title{
Fabrication of Micropatterns by Laser Direct Writing Using Nanomaterials
}

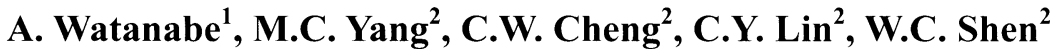 \\ ${ }^{1}$ Institute of Multidisciplinary Research for Advanced Materials, \\ Tohoku University, 2-1-1 Katahira, Aoba-ku, Sendai 980-8577, Japan \\ ${ }^{2}$ ITRI South, Industrial Technology Research Institute, No. 8, Gongyan Rd. \\ LiuJia Shiang, Tainan City 734, Taiwan, R.O.C.
}

Keywords: laser direct writing, laser sintering, micropatterning, Cu nanoparticle-dispersed ink, micro-Raman spectroscopy, femtosecond laser

\section{Introduction}

Laser direct writing method using a soluble nanoparticle provides a new process for the fabrication of micropatterns by a wet process [1-5]. One of the most interesting and important issues is the fabrication of $\mathrm{Cu}$ micropatterns by laser direct writing using a $\mathrm{Cu}$ nanoparticle ink. The copper interconnection is one of the most important technologies in microelectronics. The sintering and metallization processes are illustrated schematically in Fig.1, where the laser sintering process of a $\mathrm{Cu}$ nanoparticle ink is compared to that using a conventional electric furnace. The thermal decomposition of organic moieties which are stabilizing $\mathrm{Cu}$ nanoparticle surface induces the sintering of $\mathrm{Cu}$ nanoparticles. The surface oxidation is a compet- ing process to the $\mathrm{Cu}$ nanoparticle sintering. In the case of a slow thermal process by heat treatment using an electric furnace, the formation of copper oxide increases the resistivity. On the other hand, the residue carbon also increases the resistivity in the case of the heat treatment in an inert gas or in vacuo. It is expected that the surface oxidation can be reduced by a fast sintering process based on the laser irradiation. In this paper, we report the laser direct writing of $\mathrm{Cu}$ micropatterns by a continuous wave $(\mathrm{CW})$ laser and a high repetition rate femtosecond laser. The surface oxidation of the $\mathrm{Cu}$ nanoparticle during sintering is expected to be decrease due to the short pulse duration of the femtosecond laser.

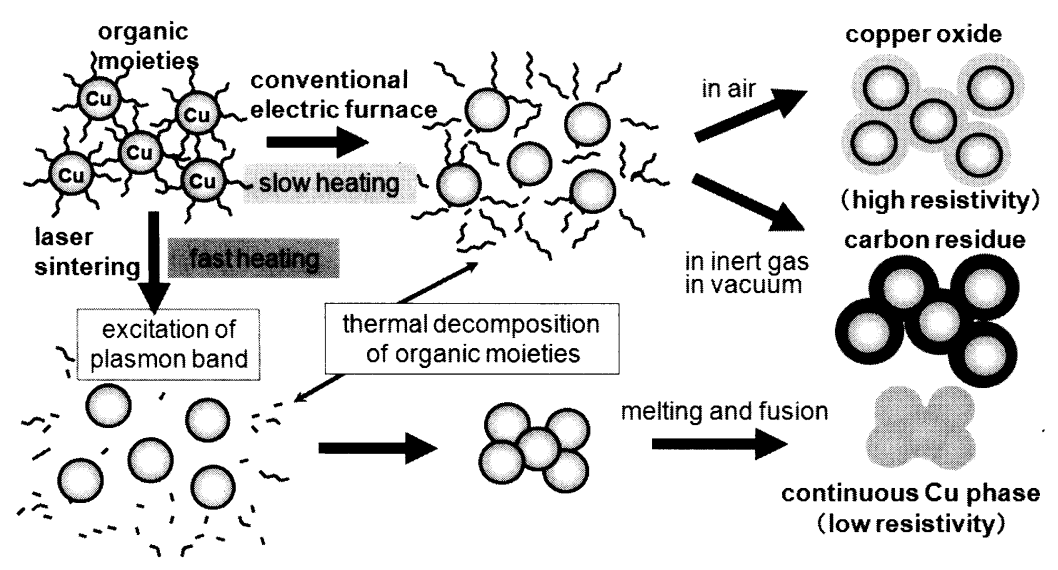

Fig.1. Sintering processes of $\mathrm{Cu}$ nanoparticles.

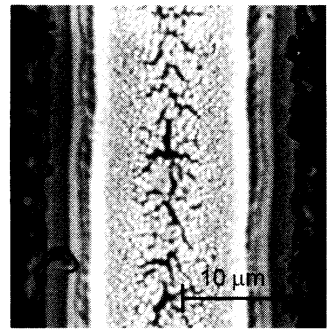

Fig.2. Optical microphotograph of $\mathrm{Cu}$ microline prepared by laser direct writing using $\mathrm{Cu}$ nanoparticle-dispersed ink in air. Laser: Ar ion laser, power density: $3.18 \mathrm{~kW} / \mathrm{cm}^{2}$, scan rate: 100 $\mu \mathrm{m} / \mathrm{s}$. 


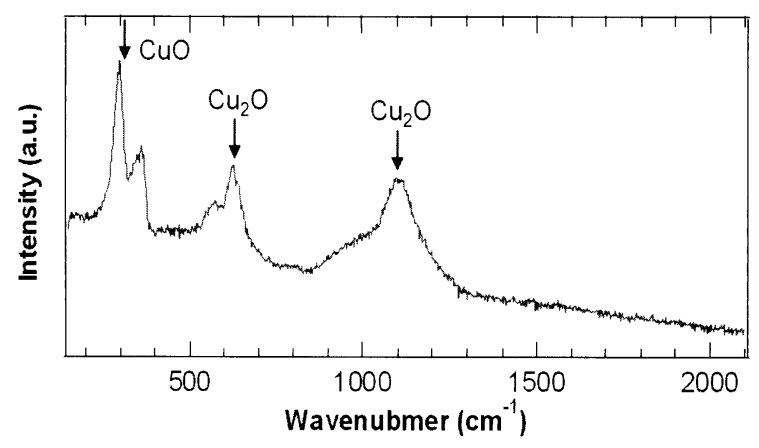

Fig.3. Micro-Raman spectrum of $\mathrm{Cu}$ microline surface. Laser: Ar ion laser $(488 \mathrm{~nm})$, power density: $3.18 \mathrm{~kW} / \mathrm{cm}^{2}$, scan rate: $100 \mu \mathrm{m} / \mathrm{s}$.

\section{Experimental}

$\mathrm{A} \mathrm{Cu}$ nanometal ink (Cu1T) was purchased from ULVAC Technologies Inc. A $\mathrm{Cu}$ nanoparticle dispersed film was prepared by spin-coating on a glass substrate. An Ar ion laser (wavelength: 488 and $514.5 \mathrm{~nm}$, MELLES GRIOT 543-300A, 300 $\mathrm{mW}$ ) was used as a CW laser source. A Ti:Sappire laser $(800 \mathrm{~nm}, 80 \mathrm{MHz}$, pulse duration $\sim 100 \mathrm{fs}$, Spectra-Physics Mai-Tai) was used as a femtosecond laser source. The focused laser beam was irradiated on the $\mathrm{Cu}$ nanoparticle dispersed film and then scanned by a PC-controlled $x-y-z$ stage system. After the irradiation of laser beam, the etching of unirradiated area using toluene gave $\mathrm{Cu}$ micropatterns. The surface chemical structure was investigated by micro-Raman spectroscopy.

\section{Results and discussion}

The optical micrograph of the $\mathrm{Cu}$ microline prepared by $\mathrm{CW}$ laser direct writing in air is shown in Fig.2, where the scan rate of the CW laser beam is $100 \mu \mathrm{m} / \mathrm{s}$. The micrograph shows rough surface morphology. Especially, the center of the line, where the power density of the Gaussian laser beam is maximum, shows inhomogeneous granular structure. The chemical structure of the rough surface was investigated by micro-Raman spectroscopy. The Raman bands assigned to copper oxide around $300 \mathrm{~cm}^{-1}$ and cuprous oxide around 600 and $1100 \mathrm{~cm}^{-1}$ were observed as shown in Fig.3. The inhomogeneous granular structure of the $\mathrm{Cu}$ microline surface and the surface oxidation could be reduced with increasing the laser scan rate. The excess duration of the laser beam causes the surface oxidation of the $\mathrm{Cu}$ microline in air. The cooling and solidification of the sintering area must be delayed by a long duration of laser beam, which enhances the oxygen diffusion and causes the oxidation. The optimization of the heating and

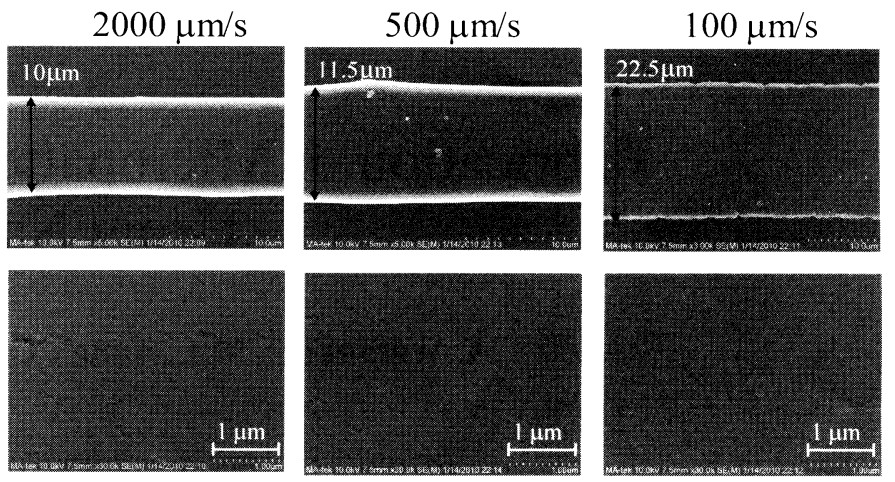

Fig.4. SEM photographs of $\mathrm{Cu}$ microlines prepared by femtosecond laser $(800 \mathrm{~nm}, 80 \mathrm{MHz})$ sintering. $150 \mathrm{~mW}$.

cooling rates must be important to reduce the oxidation of the sintered $\mathrm{Cu}$ layer. The heating and cooling rates can be controlled by changing laser scan rate. The application of a pulse laser with a high repetition rate must be also effective to control the sintering process. In the case of the pulse laser irradiation, the sintering and the ablation processes are competitive. A laser with a high repetition rate is advantageous to the sintering process because of the heat accumulation effect [6]. Fig.4 shows the SEM photographs of $\mathrm{Cu}$ microlines prepared by femtosecond laser sintering. A homogeneous $\mathrm{Cu}$ microline was prepared by the femtosecond laser sintering even at the slow scan rate of $100 \mu \mathrm{m} / \mathrm{s}$. The homogeneity was enhanced with decreasing the scan rate as shown in Fig. 4. The decrease of the line width was also observed with increasing the scan rate, which can be attributed to the decrease of the thermal diffusion from the laser beam spot.

In conclusion, the femtosecond laser irradiation with a high repetition rate is effective to inhibit the surface oxidation during the sintering of $\mathrm{Cu}$ nanoparticles in air.

\section{References}

1. A. Watanabe, Y. Kobayashi, M. Konno, S. Yamada, T. Miwa, Jpn J. Appl. Phys., 44(23), L740 (2005).

2. A. Watanabe and T. Miyashita, J. Photopolym. Sci. Technol., 20(1) 115 (2007).

3. M. Aminuzzaman, A. Watanabe and $T$. Miyashita, J. Mater. Chem., 18, 5092 (2008).

4. M. Aminuzzaman, A. Watanabe and T. Miyashita, Thin Solid Films, 517, 5935 (2009)

5. A. Watanabe, M. Aminuzzaman, and T. Miyashita, Proc. SPIE, 7202, 720206 (2009).

6. C.W. Cheng et al, Journal of Laser Micro/Nanoengineering, 4 (2009). 